\section{Impacto del delirium en pacientes de edad avanzada hospitalizados: un estudio prospectivo de cohortes}

\author{
MARCELA CARRASCO ${ }^{1, *}$, LUIGI ACCATINO-SCAGLIOTTI ${ }^{2}$, \\ JORGE CALDERÓN², LUIS VILLARROEL ${ }^{3, a}$, \\ PEDRO PAULO MARÍN', MATÍAS GONZÁLEZ ${ }^{2, a, *}$
}

\section{Delirium in older medical inpatients. A one year follow up study}

Background: Delirium is an important problem in older medical inpatients. Aim: To assess if delirium is associated with higher mortality, functional decline or higher rates of readmission or institutionalization in a one year follow-up period. Material and Methods: Prospective cohort study of consecutive patients 65 years and older, admitted to a general hospital medical ward. A psychogeriatric team assessed patients every $48 \mathrm{~h}$ using the Confusion Assessment Method (CAM), functionality, acute severity and comorbidity scores. Analysis of one year mortality and telephone functional assessment was performed. Results: Five hundred forty two patients were enrolled and $35.4 \%$ had delirium. After one year, mortality was 34.9 and $13 \%$ in delirium and non-delirium cohorts, respectively $(p<0.01)$. After adjustment for covariates, delirium was independently associated with higher mortality, and higher functional decline and institutionalization. No significant differences were seen in readmission rates. Conclusions: Delirium was significantly associated with higher mortality and functional decline over a one year follow up period in geriatric inpatients.

(Rev Med Chile 2012; 140: 847-852).

Key words: Aged; Delirium; Mortality; Prognosis.

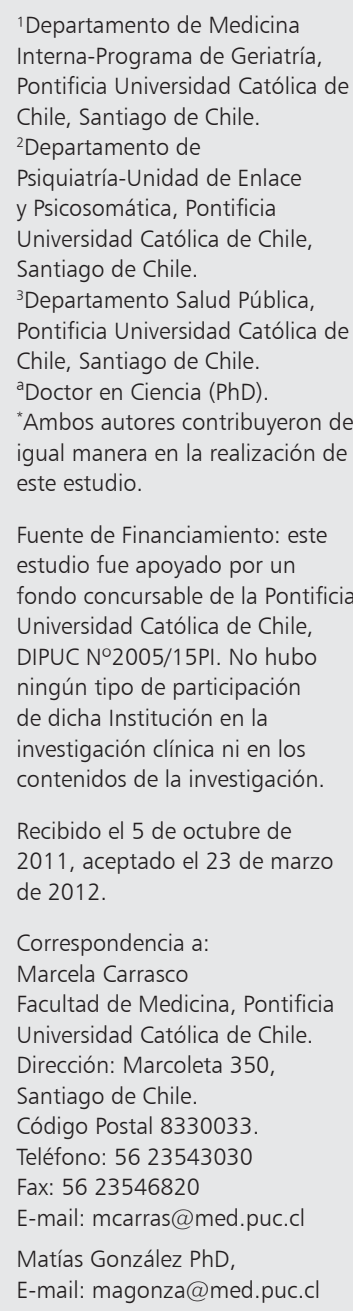

Correspondencia a: Marcela Carrasco Facultad de Medicina, Pontificia Universidad Católica de Chile. Dirección: Marcoleta 350,

Santiago de Chile.

Código Postal 8330033.

Teléfono: 5623543030

Fax: 5623546820

E-mail: mcarras@med.puc.cl

Matías González PhD,

E-mail:magonza@med.puc.cl

$\mathrm{E}$ 1 delirium es un síndrome caracterizado por alteración de la conciencia, de la atención y del pensamiento, que se inicia en forma aguda y presenta un curso fluctuante, en el que pueden aparecer, además, ideas delirantes y alucinaciones ${ }^{1}$. Su prevalencia en pacientes hospitalizados aumenta con la edad y varía según el diagnóstico de ingreso. Es considerado el segundo síndrome psiquiátrico más prevalente en el ámbito hospitalario después de los trastornos del ánimo ${ }^{2}$ afectando de 11 a 42\% de los pacientes ingresados ${ }^{3}$ y hasta $80 \%$ de los pacientes de unidades de cuidados intensivos ${ }^{4}$. Aún no existe una comprensión cabal de su fisiopatología, sin embargo, se reconoce una alteración funcional de niveles de neurotransmisores, hiperactividad del eje hipotálamo-hipófisis-glándula adrenal y aumento en la producción de citoquinas ${ }^{5}$.

La presencia de delirium ha demostrado estar asociada a mal pronóstico, aumentar las complicaciones intrahospitalarias, tales como infecciones, caídas, incontinencia y úlceras de decúbito ${ }^{6,7}$, provocando estadías hospitalarias significativamente más prolongadas y generando elevados costos en salud $^{8-10}$. Sin embargo, las consecuencias no terminan con el alta del paciente, y se ha demostrado asociación entre delirium y mayor riesgo de institucionalización, demencia y mortalidad, en forma independiente a importantes variables como edad, sexo, comorbilidades, severidad de enfermedad y deterioro cognitivo basal ${ }^{11}$. 
El delirium es considerado un marcador de estado del paciente geriátrico hospitalizado ${ }^{12}$, ya que los pacientes que lo padecen presentarían un incremento en la complejidad desde el punto de vista de su manejo individual y de lo que implica para los servicios asistenciales. Por otro lado, al ser potencialmente prevenible, puede ser considerado un indicador de calidad asistencial.

Existen pocos estudios prospectivos de pacientes mayores que cursan con delirium en Latinoamérica, el objetivo de este estudio es aportar más información sobre el pronóstico de los pacientes que han cursado con delirium durante su estadía hospitalaria, específicamente su asociación con mortalidad y el deterioro funcional a largo plazo, medidos a un año.

\section{Material y Métodos}

Estudio desarrollado en el Servicio de Medicina del Hospital Clínico de la Pontificia Universidad Católica de Chile, hospital que cuenta con 536 camas y cubre una extensa área de la ciudad de Santiago de Chile. El protocolo de estudio fue aprobado por el Comité de Ética de la institución y se obtuvo consentimiento informado de los pacientes o sus representantes previo a su ingreso al estudio.

Los participantes fueron pacientes con al menos 65 años de edad, ingresados en forma consecutiva, hospitalizados en las últimas 48 horas, en el servicio de medicina del hospital entre marzo y octubre del año 2006. Los criterios de exclusión utilizados fueron la presencia de afasia severa, coma e incapacidad para participar de la entrevista clínica.

\section{Protocolo de estudio}

Los pacientes fueron enrolados cada 48 horas por un equipo compuesto por psico-geriatras entrenados en la detección de delirium. Se consignaron los datos demográficos (sexo, edad, RUN, información de contacto) y se les aplicaron escalas de evaluación: Acute Physiology and Chronic Health Evaluation II (APACHE II), que permite medir la severidad de la enfermedad aguda mediante el uso de datos clínicos y de laboratorio ${ }^{13}$, el Índice de Comorbilidad de Charlson, que estima el grado de comorbilidad de los pacientes ${ }^{14}$, el nivel de funcionamiento previo fue medido mediante el Indice de Barthel ${ }^{15}$, que mide la capacidad de los pacientes para realizar 10 actividades básicas de la vida diaria con puntajes que van desde 0 (funcionalidad mínima) a los 100 puntos (funcionalidad óptima), y por último se aplicó el Cuestionario de Evaluación Funcional de Pfeffer (PFAQ) ${ }^{16}$ que estima funcionalidad basal mediante información otorgada por el cuidador, en que puntajes sobre 7 sugieren la presencia de demencia previa ${ }^{17}$.

La presencia de delirium fue determinada mediante el Confusion Assesment Method (CAM) ${ }^{18}$, en su versión adaptada al español ${ }^{19}$. El CAM es un instrumento validado para detectar delirium y está basado en la aplicación operacional de los criterios diagnósticos del DSM III-R, obteniendo la información relevante desde el paciente y su cuidador. Para diagnosticar delirium sigue un algoritmo diagnóstico basado en la presencia de dos criterios principales: 1) inicio agudo y curso fluctuante y; 2) inatención, y la presencia de al menos uno de los dos criterios secundarios: 1) pensamiento desorganizado; y 2) compromiso de conciencia.

\section{Procedimiento}

El equipo de investigadores evaluó mediante $\mathrm{CAM}^{19}$ la presencia y duración del delirium en los pacientes enrolados cada 48 horas. La evaluación se realizó entre las 9 AM y las 2 PM hasta el alta o por un máximo de 12 días. La información necesaria para el APACHE II ${ }^{13}$ y el Índice de Comorbilidad de Charlson ${ }^{14}$ fue obtenida de los registros médicos y de enfermería. Se entrevistó a los cuidadores de los pacientes para evaluar la funcionalidad mediante los Indices de Barthel ${ }^{15}$ y Pfeffer $^{16}$. En estas entrevistas se preguntó sobre la capacidad de los pacientes para realizar las actividades de la vida diaria dos semanas previas al ingreso hospitalario. Se registraron los datos de exámenes del laboratorio de las primeras $24 \mathrm{~h}$, específicamente nitrógeno ureico (BUN), creatinina plasmática, electrolitos plasmáticos, proteína $C$ reactiva $(\mathrm{PCR})$, velocidad de eritrosedimentación (VHS), albúmina y hemoglobina plasmáticas.

En caso de que el paciente evaluado presente un tamizaje CAM positivo, en la evaluación inicial o en las de seguimiento durante la estadía hospitalaria, el paciente era incluido en la cohorte de delirium. Si no se diagnosticaba delirium el paciente ingresaba a la cohorte sin delirium. Se determinó la mortalidad de acuerdo a datos del Registro Civil de Identificación Chileno, usando los registros electrónicos en base al RUN y la funcionalidad después de 12 meses mediante en- 
trevistas telefónicas estructuradas, así como para obtener información de institucionalización o rehospitalización en el período.

El equipo de investigadores no participó en los cuidados hospitalarios ni ambulatorios de los pacientes.

\section{Estadística}

Los datos fueron analizados mediante el programa estadístico SPSS 15.0. Para el análisis bivariado de variables cualitativas y proporciones se utilizó la prueba de $\chi^{2}$ y las variables cuantitativas fueron analizadas a través de la prueba $T$ para igualdad de medias. Se consideró significativo un $\mathrm{p}<0,001$. Con el fin de evaluar el impacto del delirium en la mortalidad al año, se realizó un modelo de regresión logística ${ }^{20}$, controlando por las posibles variables confundentes incluyendo en el modelo: edad, género, puntaje APACHE, puntajes de Barthel basal, índice de Charlson, valor de Pffefer mayor de 7, el valor de albúmina y hemoglobina basal.

\section{Resultados}

Un total de 542 pacientes fueron ingresados al estudio. El promedio de edad fue de 78 años y su estadía promedio en el hospital fue de 5,8 \pm 4,8 días. El 62\% de los pacientes fueron mujeres. Los diagnósticos médicos primarios de la muestra se agruparon de la siguiente manera: patologías infecciosas (31\%), alteraciones metabólicas (15\%), patologías gastrointestinales (14\%), patologías cardiovasculares $(10,5 \%)$ y otras patologías $(30 \%)$.

Se pesquisó delirium en 192 pacientes (35,4\%), la mayoría $(87 \%)$ durante las primeras 48 horas de hospitalización. La duración promedio del delirium fue de 4,9 días $\pm 3,1$. Según se muestra en la Tabla 1, la cohorte con delirium era de mayor edad (81,5 \pm 7,2 años versus 75,8 \pm 7,0 años), con una funcionalidad basal más deteriorada, de acuerdo al Índice de Barthel más bajo (73,8 \pm 24,3 versus $92,7 \pm 15,1)$ y un puntaje de Pfeffer más elevado $(9,1 \pm 9,7$ en cohorte con delirium versus $2,2 \pm$ $5,1)$. En cuanto a gravedad de la enfermedad aguda según APACHE la cohorte con delirium presentó un puntaje mayor, como asimismo, en exámenes de laboratorio del ingreso valores de albumina más baja y mayor valores de nitrógeno ureico $(\mathrm{p}<0,05)$, sin diferencias significativas en otras variables como hemoglobina, creatinina, natremia, velocidad de sedimentación (VHS), proteína C reactiva $(\mathrm{PCR})$ ni hemoglobina.

La cohorte con delirium presentó mayor estadía

Tabla 1. Características basales de la población estudiada, según delirium

\begin{tabular}{|c|c|c|c|c|}
\hline & $\begin{array}{l}\text { Grupo total } \\
(n=542) \\
\text { Prom** (DE) }\end{array}$ & $\begin{array}{l}\text { Cohorte con } \\
\text { delirium }(n=350) \\
\text { Prom }^{* *}(D E)\end{array}$ & $\begin{array}{c}\text { Cohorte sin } \\
\text { delirium }(n=192) \\
\text { Prom }^{* *}(\mathrm{DE})\end{array}$ & Valor $\mathbf{p}^{*}$ \\
\hline Edad (años) & $77,9(7,6)$ & $81,5(7,2)$ & $75,8(7,0)$ & $<0,001$ \\
\hline APACHE II puntaje & $10,1(4,2)$ & $10,7(4,3)$ & $9,7(4,1)$ & 0,006 \\
\hline Índice Charlson & $1,7(1,6)$ & $1,8(1,6)$ & $1,6(1,6)$ & 0,141 \\
\hline Índice Barthel & $86,0(20,9)$ & $73,8(24,3)$ & $92,7(15,1)$ & $<0,001$ \\
\hline Índice Pfeffer & $4,6(7,8)$ & $9,1(9,8)$ & $2,2(5,0)$ & $<0,001$ \\
\hline Estadía hospitalaria (d) & $5,8(4,8)$ & $7,3(5,9)$ & $5,0(3,9)$ & $<0,001$ \\
\hline Sodio (mEq/L) & $137,8(5,9)$ & $137,2(7,7)$ & $138,1(4,6)$ & 0,159 \\
\hline Creat $(\mathrm{mg} / \mathrm{dL})$ & $1,4(1,5)$ & $1,5(1,4)$ & $1,4(1,6)$ & 0,599 \\
\hline $\mathrm{BUN}(\mathrm{mg} / \mathrm{dL})$ & $26,9(19,7)$ & $31,8(22,4)$ & $24,1(17,4)$ & $<0,001$ \\
\hline Albumina (mg/dL) & $3,7(1,8)$ & $3,5(0,6)$ & $3,9(2,2)$ & 0,003 \\
\hline Hemoglobina (g/dL) & $12,8(2,3)$ & $12,6(2,2)$ & $12,9(2,4)$ & 0,176 \\
\hline $\mathrm{VHS}^{* * *}$ & $35,5(28,7)$ & $36,9(28,8)$ & $34,7(28,6)$ & 0,392 \\
\hline$P C R^{* * * *}$ & $7,2(8,8)$ & $6,8(8,2)$ & $7,4(9,1)$ & 0,487 \\
\hline
\end{tabular}

*Análisis de varianza y Pruebas t de Student para variables continuas. $\chi^{2}$ para comparar variables categóricas y dicotómicas. **Promedio. ***Velocidad de eritrosedimentación. ****Proteína $C$ reactiva (valor normal $<1,0$ ). DE: Desviación estándar. 
Impacto del delirium en pacientes de edad avanzada hospitalizados - M. Carrasco et al

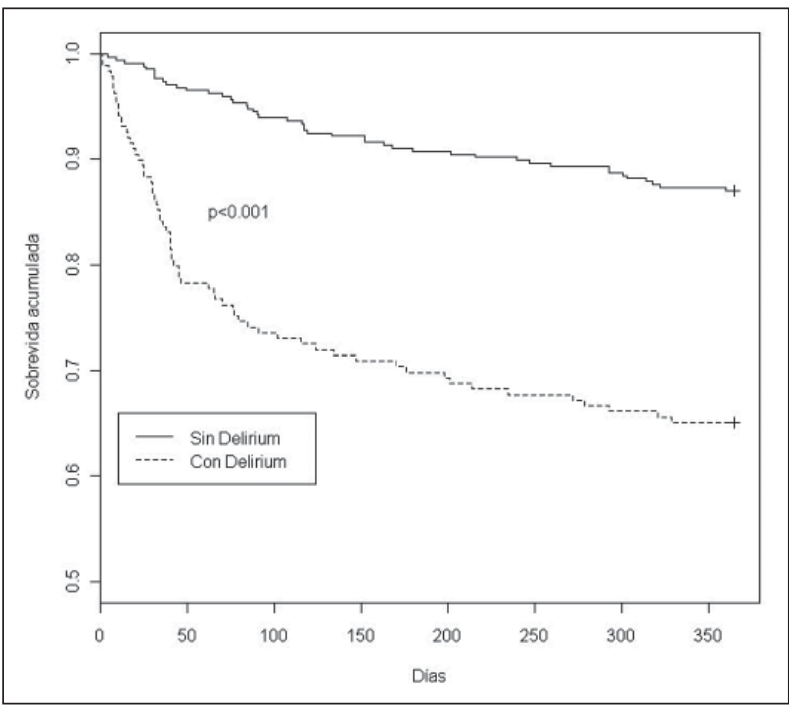

Figura 1. Sobrevida al año según presencia de Delirium.

hospitalaria $(7,3 \pm 5,9$ días versus $5,0 \pm 3,9$ días), así como una mayor mortalidad durante la hospitalización $8,5 \%$ versus $1,7 \%$ en la cohorte sin delirium.

\section{Seguimiento al año}

De los 542 pacientes, seis $(1,1 \%)$ fueron perdidos de seguimiento a los 12 meses, la mortalidad global fue de 20,7\% (111 de 536 pacientes). La mortalidad en la cohorte con delirium fue $34,9 \%$, versus $13,0 \%$ en la cohorte sin delirium (RR 2,71 [CI 95\% 1,96-3,75]) (Figura 1).

$\mathrm{Al}$ realizar el análisis de regresión logística con el fin de evaluar el peso de la variable delirium sobre la mortalidad a un año, se encontró que las variables independientemente asociadas a morir antes del año de seguimiento fueron delirium, Índice de Comorbilidad de Charlson y la albúmina basal.
$\mathrm{Al}$ ajustar el resultado por las demás variables analizadas en el estudio (edad, Índice de Comorbilidad de Charlson, APACHE II, Índice de Barthel, Pfeffer, además de albúmina), la presencia de delirium se asoció en forma independiente a mayor mortalidad.

En relación con la funcionalidad se observó que la cohorte con delirium, se deterioró en forma significativa tanto en la medición según Barthel como en el Pfeffer $(\mathrm{p}<0,001)$, como se muestra en la Tabla 2. En este mismo sentido, fue más frecuente en la cohorte con delirium la presencia de sospecha de demencia según Pfeffer mayor de 7, utilizado como aproximación a deterioro cognitivo ${ }^{17}$.

En frecuencia de institucionalización, se observó que 9 pacientes de la cohorte con delirium fueron ingresados a residencias de larga estadía o institucionalizados versus 1 paciente de la cohorte sin delirium $(\mathrm{p}<0,001)$. No hubo diferencias en el número de rehospitalizaciones.

\section{Discusión}

Los resultados de este estudio apoyan, con una cohorte de gran de tamaño, la elevada incidencia y prevalencia de delirium en los pacientes ancianos hospitalizados de un hospital universitario. Se confirma la asociación entre delirium y edad avanzada, severidad de enfermedad aguda, peor funcionalidad basal y deterioro cognitivo.

Se identifica al delirium como un factor de riesgo independiente de mortalidad,

Tabla 2. Características de las cohortes al año de seguimiento según delirium

\begin{tabular}{|lcccc|}
\hline & Grupo total & $\begin{array}{c}\text { Cohorte con } \\
\text { delirium }\end{array}$ & $\begin{array}{c}\text { Cohorte sin } \\
\text { delirium }\end{array}$ & Valor p \\
\hline Mortalidad al año, N/total (\%) & $111 / 536(20,7)$ & $66 / 189(34,9)$ & $45 / 347(13)$ & $<0,001$ \\
Índice Pfeffer (promedio (DE)/total) & $5,1(8,7) / 317$ & $12,1(10,8) / 94$ & $2,1(5,4) / 223$ & $<0,001$ \\
\hline Pfeffer $>7^{*}$, N/total (\%) & $71 / 316(22,5)$ & $51 / 94(54,3)$ & $20 / 222(9)$ & $<0,001$ \\
Índice Barthel (promedio (DE)/total) & $86,5(23,5) / 317$ & $72,7(31,8) / 94$ & $92,3(15,6) / 223$ & $<0,001$ \\
\hline Rehospitalización, N/total (\%) & $70 / 321(21,8)$ & $20 / 96(20,8)$ & $50 / 225(22,2)$ & 0,78 \\
\hline Institucionalización, N/total (\%) & $10 / 321(3,1)$ & $9 / 97(9,3)$ & $1 / 224(4,0)$ & $<0,001$ \\
\hline
\end{tabular}

* Aproximación de deterioro cognitivo ${ }^{17}$. 
asociándose a una cifra cuatro veces mayor de mortalidad intra-hospitalaria, tendencia que se mantiene en el seguimiento, en forma similar a lo observado por McCusker et $\mathrm{al}^{21}$ en un seguimiento de un año. La mayor mortalidad se mantiene incluso al ser ajustado por variables como edad, estado funcional basal, severidad de enfermedad aguda y comorbilidad. Estos resultados van en la misma línea de otros estudios previamente publicados y concuerdan con los obtenidos por Rockwood et $\mathrm{a}^{6,22,23}$. Un meta-análisis reciente confirma la asociación entre delirium y mal pronóstico, con mayor riesgo de mortalidad, institucionalización y demencia ${ }^{11}$. Los estudios que han fallado en demostrar estas asociaciones podrían carecer de un poder estadístico significativo o poseer cohortes con pocos casos de delirium ${ }^{24,25}$.

Nuestro seguimiento confirma también, la asociación del delirium con deterioro en la capacidad funcional a un año de seguimiento. Esto es muy relevante considerando que el objetivo primordial en la atención de personas mayores debe ir focalizado a la mantención de la funcionalidad y prevención de la dependencia, ya que son estas variables las que mejor de correlacionan con calidad de vida y pronóstico vital ${ }^{26}$.

La metodología de este estudio posee destacables fortalezas. El tamizaje de delirium fue realizado por un equipo de psicogeriatría no involucrado en los cuidados del paciente, con una frecuencia de $48 \mathrm{~h}$ durante la hospitalización lo que a nuestro entender es fundamental en el estudio del delirium, dada su condición característica de fluctuación sintomática, disminuyendo así la posibilidad de falsos negativos. La muestra de pacientes analizados fue de gran tamaño, con un bajo porcentaje de pérdida de seguimiento $(1,1 \%)$, lo que le otorga un adecuado poder estadístico. El análisis de los resultados se llevó a cabo controlando variables confundentes, como el grado severidad de enfermedad, comorbilidades y estado funcional basal del paciente. Nuestros resultados podrían ser extrapolables a poblaciones similares, es decir, pacientes adultos mayores ingresados en servicios de medicina, sin embargo, se requieren otros estudios que confirmen nuestros datos en otro tipo de pacientes.

Realizar estudios en población con delirium, representa un desafío importante en cuanto al diseño y seguimiento de estas cohortes. En nuestro caso gracias al sistema de Registro de Mortalidad
Nacional, nos permitió tener datos confiables con escasas pérdidas en ese aspecto del seguimiento, sin embargo, esto no ocurrió en el seguimiento de la evaluación de funcionalidad y datos de rehospitalizaciones, en que dependimos del contacto telefónico del cuidador, no siempre posible y afectado por el sesgo del recuerdo. Aun así, se observa una tendencia que apoya los hallazgos de datos previos.

El impacto de la presencia de delirium en la mortalidad reafirma la importancia de incorporar en nuestra práctica clínica al delirium como un marcador de estado de salud en el paciente hospitalizado ${ }^{12}$. Ello implica que su presencia pone en alerta sobre un grupo de pacientes que requerirán una atención especial por parte del sistema sanitario, dado su mayor riesgo de complicaciones, deterioro funcional y mortalidad tanto a corto como a largo plazo, asociándose también a mayores costos en salud.

El delirium es una condición potencialmente prevenible ${ }^{27,28}$, lo que permite considerarlo como un marcador de calidad asistencial de los servicios que manejen pacientes mayores. Así, los servicios que implementes estrategias sistematizadas en prevención, detección precoz y tratamiento eficaz del delirium, tendrán un buen indicador de calidad de atención que ofrecen a pacientes de mayor riesgo y complejidad. Hay que asumir como un hecho que los cambios demográficos aumentarán la presencia de pacientes mayores en los sistemas de salud, y ellos requieren una adaptación de los actuales sistemas sanitarios. Esto es especialmente importante en los países en vías de desarrollo, en que se debe aplicar la evidencia disponible de manera costoefectiva $^{28,29}$. En este sentido el delirium representa un desafío en cuanto a que la implementación de protocolos logra disminuir la prevalencia de esta condición y mejorar la calidad asistencial.

\section{Referencias}

1. Lipowski ZJ. Delirium (acute confusional states). JAMA 1987; 258: 1789-92.

2. Lipowski ZJ. Delirium in the elderly patient. N Engl J Med 1989; 320: 578-82.

3. Siddiqi N, House AO, Holmes JD. Occurrence and outcome of delirium in medical in-patients: a systematic literature review. Age Ageing 2006; 35: 350-64.

4. Ely EW, Shintani A, Truman B, Speroff T, Gordon SM, Harrell FE, Jr. et al. Delirium as a predictor of mortality 
in mechanically ventilated patients in the intensive care unit. JAMA 2004; 291: 1753-62.

5. Young J, Inouye SK. Delirium in older people. BMJ 2007; 334: 842-6.

6. Francis J. Delirium in older patients. J Am Geriatr Soc 1992; 40: 829-38.

7. Tinetti ME. Clinical practice. Preventing falls in elderly persons. N Engl J Med 2003; 348: 42-9.

8. Franco K, Litaker D, Locala J, Bronson D. The cost of delirium in the surgical patient. Psychosomatics 2001; 42: 68-73.

9. Leslie DL, Marcantonio ER, Zhang Y, Leo-Summers L, Inouye SK. One-year health care costs associated with delirium in the elderly population. Arch Intern Med 2008; 168: 27-32.

10. González M, Uslar W, Villarroel L, Calderón J, Palma C, Carrasco M. [Hospital costs associated with delirium in older medical patients]. Rev Esp Geriatr Gerontol 2012; 47 (1): 23-6.

11. Witlox J, Eurelings LS, de Jonghe JF, Kalisvaart KJ, Eikelenboom P, van Gool WA. Delirium in elderly patients and the risk of postdischarge mortality, institutionalization, and dementia: a meta-analysis. JAMA 2010; 304 : 443-51.

12. González M, Carrasco M. [Delirium: a marker of health status in the geriatric patient]. Rev Esp Geriatr Gerontol 2008; 43 Suppl 3: 38-41.

13. Knaus WA, Draper EA, Wagner DP, Zimmerman JE. APACHE II: a severity of disease classification system. Crit Care Med 1985; 13: 818-29.

14. Deyo RA, Cherkin DC, Ciol MA. Adapting a clinical comorbidity index for use with ICD-9-CM administrative databases. J Clin Epidemiol 1992; 45: 613-9.

15. Mahoney FI, Barthel DW. Functional Evaluation: The Barthel Index. Md State Med J 1965; 14: 61-5.

16. Pfeffer RI, Kurosaki TT, Harrah CH, Jr., Chance JM, Filos S. Measurement of functional activities in older adults in the community. J Gerontol 1982; 37: 323-9.

17. Juva K, Makela M, Erkinjuntti T, Sulkava R, Ylikoski R, Valvanne J, et al. Functional assessment scales in detecting dementia. Age Ageing 1997; 26: 393-400.

18. Inouye SK, van Dyck CH, Alessi CA, Balkin S, Siegal
AP, Horwitz RI. Clarifying confusion: the confusion assessment method. A new method for detection of delirium. Ann Intern Med 1990; 113: 941-8.

19. González M, de Pablo J, Fuente E, Valdés M, Peri JM, Nomdedeu M, Matrai S. Instrument for detection of delirium in general hospitals: adaptation of the confusion assessment method. Psychosomatics 2004; 45: 426-31.

20. Hosmer D, Lemeshow S. Applied logistic regression. New York: Wiley-Interscience, 2000.

21. McCusker J, Cole M, Abrahamowicz M, Primeau F, Belzile E. Delirium predicts 12-month mortality. Arch Intern Med 2002; 162: 457-63.

22. Rockwood K, Cosway S, Carver D, Jarrett P, Stadnyk K, Fisk J. The risk of dementia and death after delirium. Age Ageing 1999; 28: 551-6.

23. Leslie DL, Zhang Y, Holford TR, Bogardus ST, LeoSummers LS, Inouye SK. Premature death associated with delirium at 1-year follow-up. Arch Intern Med 2005; 165: 1657-1662.

24. Adamis D, Treloar A, Darwiche FZ, Gregson N, Macdonald AJ, Martin FC. Associations of delirium with in-hospital and in 6-months mortality in elderly medical inpatients. Age Ageing 2007; 36: 644-9.

25. Inouye SK, Rushing JT, Foreman MD, Palmer RM, Pompei P. Does delirium contribute to poor hospital outcomes? A three-site epidemiologic study. J Gen Intern Med 1998; 13: 234-42.

26. Freedman V, Martin L, Schoeni R. Recent trends in disability and functioning among older adults in the United States. A systematic review. JAMA 2002; 288: 3137-46.

27. Inouye SK, Schlesinger MJ, Lydon TJ. Delirium: a symptom of how hospital care is failing older persons and a window to improve quality of hospital care. Am J Med 1999; 106: 565-73.

28. O’Mahony R, Murthy L, Akunne A, Young J. Synopsis of the National Institute for Health and Clinical Excellence Guideline for Prevention of Delirium. Ann Intern Med 2011; 154: 746-51.

29. Marcantonio ER, Flacker JM, Wright RJ, Resnick NM. Reducing delirium after hip fracture: a randomized trial. J Am Geriatr Soc 2001; 49: 516-22. 